\title{
Changing accessibility, dwelling price and the spatial distribution of socio-economic activities
}

\author{
Pierluigi Coppola*, Agostino Nuzzolo \\ Department of Civil Engineering, “Tor Vergata” University of Rome, Via del Politcenico 1, 00133 Roma, Italy
}

\section{A R T I C L E I N F O}

\section{Article history:}

Available online 30 December 2010

\section{Keywords:}

Land-Use/Transport Interactions (LUTI)

models

Random Utility theory

Back-casting analysis

\begin{abstract}
A B S T R A C T
In this paper we present an activities-location choice model with endogenous price which simulates, based on Expected Random Utility principle, the behaviour of several agents of the urban system (e.g. the workers distinguished by income, the firms by economic sector) to estimate the spatial distribution of socio-economic activities within the study area as well as the impact of differential changes in accessibility on the dwelling price. The study area for this research is the metropolitan area of Napoli (South Italy), for which we show the results of the model estimation and the results of a "backcasting" analysis. (C) 2010 Elsevier Ltd. All rights reserved.
\end{abstract}

\section{Introduction}

Differential changes of accessibility among the zones of a metropolitan area can have significant impacts on the land use pattern, particularly on the spatial distribution of the population and of economic activities: typically, the greater the accessibility of a zone, the greater, ceteris paribus, the number of activities located in that zone (i.e. the bigger the "location utility"). Moreover, changes in accessibility may induce changes in the local firms' productivity as well as in the dwellings market, i.e. the dwelling stock and the dwelling price. In particular, dwelling price effects arise when changes in accessibility attract new demand for houses which cannot be satisfied by the current stock. An increase in accessibility could lead to either an increase of the dwelling stock if there is room for new development or, more often, an increase of the dwelling price as the result of the interaction between dwelling demand and supply.

A number of factors contribute to the complexity of such interaction: on the one hand, the land development regulations and the decision-making processes of the land developers, which shape the dwelling supply and, in turn, affect the prices; on the other hand, the location choice behaviour of the households and of the firms which gives rise to the spatial distribution of the socioeconomic activities over the study area.

Microeconomic theory using disaggregate spatial data offers behavioural foundations and a better understanding of such decisions. Using a random utility maximization and equilibrium theory, this paper presents an activities-location choice model which

\footnotetext{
* Corresponding author. Tel.: +39 06 72597059; fax: +39 0672597053.

E-mail address: coppola@ing.uniroma2.it (P. Coppola).
}

simulates the behaviour of multiple agents of the urban system (i.e. the workers disaggregated by income, the firms by economic sector) to estimate the spatial distribution of socio-economic activities within the study area as well as the impacts on the dwellings price. The paper is organized as follows. In Section 2 we review the literature of the Land Use Transport Interaction (LUTI) models which have included (exogenously or endogenously) the simulation of the dwellings price market. In Section 3 we present the proposed LUTI modelling framework which has been developed both with endogenous and exogenous dwellings price formulations. A comparison of the results of the application to the metropolitan area of Napoli (South Italy) of the above two different modelling approaches is presented in Section 4. Finally, conclusions are drawn in Section 5.

\section{The state of the art}

Microeconomic theories of land use and transport interactions can be traced back to the early 19th century. Von Thunen first attempted to incorporate transport cost in location decisions (as reported in De la Barra, 1989). Under the assumptions of a closed system, homogenous land and zero cost of entering/leaving the agricultural market, the commodity having the highest ratio of the cost of transport (of one unit of commodity per unit of distance) to the amount of land required to produce one unit of commodity (i. e. the slope for its surplus/rent profile), takes the locations closest to the single market. Using Von Thunen's ideas, Alonso (1964) explained the urban cases, where equilibrium patterns emerge around a single employment centre. These models treat land as homogenous and continuous and recognize only one employment centre (located in the centre of an imaginary study area). 
Herbert and Stevens' (1960) model determined residential prices by maximizing aggregate rents subject to constraints on (total) land availability and the number of households to be accommodated. Such a model treats spatial elements in an aggregate manner, using an exhaustive zone-based subdivision of the region. Other simulation models rely on artificial intelligence methods, like neural networks, genetic algorithms and cellular automata (e.g. Clarke, Hoppen, \& Gaydos, 1997). These models may mimic many aspects of the dynamic and complex land use system, but they generally lack behavioural foundations to explain the process.

More advanced models (e.g. Anas \& Xu, 1999; Chang \& Mackett, 2005) predict the households distribution via a general equilibrium and land use-transportation interactions approach. However, their complexity has greatly limited their application.

More recently, the "UrbanSim" model (Waddell, 2002; Waddell et al., 2003) simulates the land-market interactions with the location choices of households, firms, developers and public actors, in a micro-simulation framework. Following this approach, Zhou and Kockelman (2008) examine microscopic equilibrium of the single-family residential land development based on the bid-rent theory. A logit model and notions of price competition are used to simulate household location choices in different scenarios, with either one or multiple employment centres and with low, medium and high value-of-travel-time assumptions. Finally De Palma, Motamedi, Picard, and Waddell (2006) coupled Urbansim with the dynamic traffic assignment model "Metropolis", developing a modelling framework which explicitly allows for two types of endogeneity in residential location choices: the interdependency of residential location with dwellings price, and the interdependency of residential location with the travel times for work trips.

Basically, the above modelling frameworks can be cast into two classes of models according to the way in which the interactions between accessibility/travel costs and dwellings price are simulated: the class of exogenous dwellings price models and the endogenous ones (Fig. 1).

The exogenous dwelling price models are based on linear multivariate regression: the average price of the houses in a zone is a linear function of zonal attributes, such as the accessibility to services, the presence of green spaces, etc. (the "hedonic price" approach).

The endogenous dwelling price models estimate the dwelling price jointly with the location of the socio-economic activities. The dwelling price in a given zone results from the interaction among demand and supply in that zone, where the demand for dwellings depends on the location utility of that given zone and, therefore, on the transport system.

The major innovation presented in this paper is the development of an endogenous price model including different urban agents (residents and firms), who have a heterogeneous behaviour not only with respect to the willingness-to-pay for renting or buying a house, as in Zhou and Kockelman (2008), but also in the typology of the dwelling unit needed; the latter implying the segmentation of the dwelling stock into different typologies available only for specific agents. In addition, for the first time, a validation on a realistic case study, by means of a backcasting analysis of the two different approaches to the simulation of the interactions between accessibility, activities, spatial distribution and dwelling price (i.e. the endogenous and the exogenous approach) is presented.

\section{The proposed modelling framework}

The aim of the proposed modelling system is to predict the spatial distribution within a given study area of the urban activities (e.g. residents, services and commercial activities) due to changes in the performances of the transportation systems, the latter affecting the zone accessibility and the generalized travel costs between zones.

We assume that the distribution of the spatial activities derives from the location choices of several urban decision makers (in the remainder of the paper these are called "agents"). For instance, the distribution of the population derives from where the households choose to live; the distribution of the services derives from where the firms decide to locate their offices.

The adopted behavioural paradigm, underlying such decision processes, is that of Random Utility Maximization (RUM). In fact, it is assumed that the urban agents choose to locate their activities (e.g. the choice of residing in a given zone, or the choice of locating an office or a shop in a given zone) in the zones which do maximize their utility (i.e. the location utility). The latter depends, in turn, on several attributes related to the characteristics of the zone itself (e.g. the dwelling price per square metre, the availability of floor space, the accessibility, etc.), and particular to the specific agent (e.g. the income, the willingness-to-pay for renting/purchasing a dwelling, and so on).

Consistently with the RUM principles we assume the utility is a random variable consisting of two terms: the systematic utility and the random residuals. Then, if the random residuals are assumed to be independently and identically Gumble distributed, the probability for the agent "i" of locating its activity in zone "o", $P^{\mathrm{i}}(\mathrm{o})$ is given by the well-known Logit formulation:

$$
P^{\mathrm{i}}(\mathrm{o})=\frac{\exp \left[\mathrm{V}^{\mathrm{i}}(\mathrm{o})\right]}{\sum \mathrm{o}^{\prime} \exp \left[\mathrm{V}^{\mathrm{i}}\left(\mathrm{o}^{\prime}\right)\right]}
$$

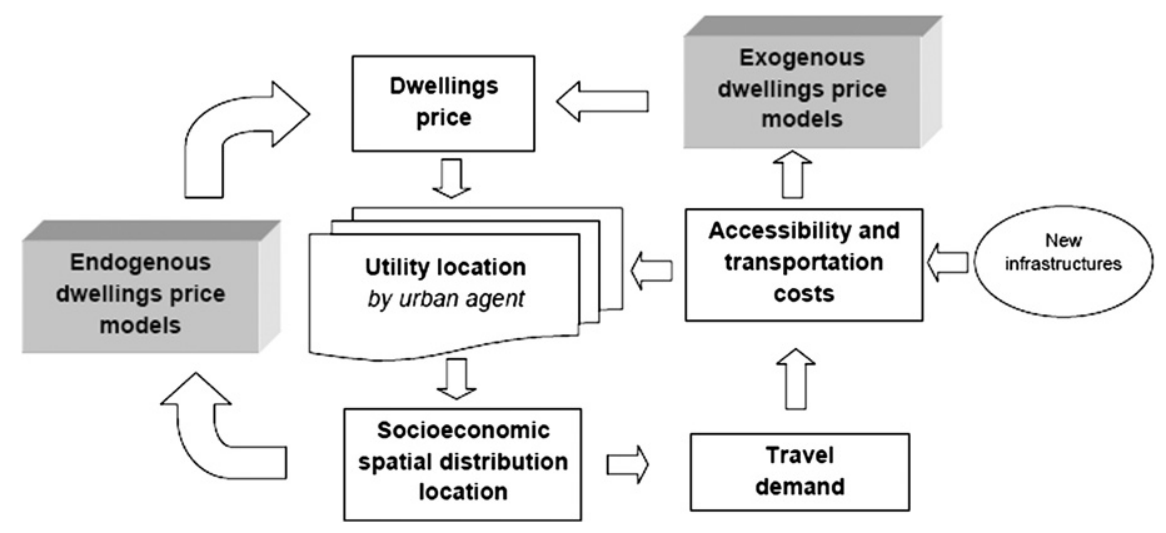

Fig. 1. Exogenous vs. endogenous dwelling price models. 
where $V^{\mathrm{i}}(\mathrm{o})$ is the systematic location utility of zone "o", relative to the agent " $\mathrm{i}$ ".

For the generic firm, the systematic utility of locating the activity in a zone is given by the linear combination of the attributes, $X_{\mathrm{k}}(\mathrm{o})$, depending both on the zone, "o", and on the agent, "i", weighted by the parameters $\beta^{\mathrm{i}}$ :

$V^{\mathrm{i}}(\mathbf{o})=\sum_{\mathrm{k}} \beta_{\mathrm{k}}^{\mathrm{i}} X_{\mathrm{k}}^{\mathrm{i}}(\mathbf{0})$

Given the total number of agents belonging to class "i", $A^{\mathrm{i}}$, the number of firms locating in the zone "o", $A^{\mathrm{i}}(\mathrm{o})$, follows as:

$A^{\mathrm{i}}(\mathbf{0})=P^{\mathrm{i}}(\mathbf{0}) \cdot A^{\mathrm{i}}$

On the other hand, for the generic worker, the systematic utility of locating the residence in a zone is given by the linear combination of the attributes, $X_{\mathrm{k}}$, depending not only on the zone, "o", and on the agent, "i", but also on the workplace zone "d". In fact, the number of workers residing in a given zone "o", is given by:

$A^{\mathrm{i}}(\mathbf{o})=\sum_{\mathrm{d}} P_{\text {res-cond }}^{\mathrm{i}}(\mathrm{o} \mid \mathrm{d}) \cdot J_{\text {tot }}^{\mathrm{i}}(\mathrm{d})$

where $P_{\text {res-cond }}^{\mathrm{i}}(\mathrm{o} \mid \mathrm{d})$ is the probability of choosing to live in zone "o" conditional on working in zone " $\mathrm{d}$ " and $J_{\text {tot }}^{\mathrm{i}}(\mathrm{d})$ represents the total number of jobs in the zone "d" available for workers of class "i".

Some of the attributes $X_{\mathrm{k}}(\mathrm{o})$ may depend, directly or indirectly, on the number of agents seeking to locate in the zone "o": for example, if the number of jobs (e.g. in services or commerce) located in a given zone is affected by the number of households in that zone; or if the number of households depends on the number of services located in the zone (e. g. the presence of schools or shops).

Let $x_{k}[\cdot]$ denote a function, specific to the given attribute " $k$ ", which relates the attribute to the number of agents located in the zone "o":

$X_{\mathrm{k}}(\mathbf{0})=x_{k}\left[A^{1}(\mathbf{0}), A^{2}(\mathbf{0}), \ldots, A^{n}(\mathbf{0})\right]$

This can be a linear function of one agent class, but also a more complex function of different agent classes, as we have assumed for the dwelling price attribute in the application presented in the next section. In any case, there is a circular dependency among the number of agents in the zones and the attributes of the location utility, which gives rise to an equilibrium problem. This can be treated as a fixed-point problem, whose solution is represented by the vectors $A^{\mathrm{i} *}$ and $X_{\mathrm{k}}^{*}$ :

$$
\left\{\begin{array}{l}
A^{\mathrm{i} *}(\mathrm{o})=P^{\mathrm{i}}\left[\ldots, X_{k}^{*}(\mathrm{o}), \ldots\right] \cdot A^{\mathrm{i}} \quad \forall \mathrm{i} \\
X_{k}^{*}(\mathrm{o})=x_{k}\left[\ldots, A^{\mathrm{i}^{*}}(\mathrm{o}), \ldots\right] \quad \forall k \in K
\end{array}\right.
$$

where $K$ is the subset of the attributes which depend on agents located in the zone.

The existence and the uniqueness of the equilibrium solution can be proved under certain condition of the probability functions $P$ $[\cdot]$ and of the functions $x[\cdot]$ following the conditions imposed by Brouwers' theorem (Cascetta, 2009). The uniqueness of the solution can be proved when the functions $x[\cdot]$ are strictly monotone and the location choice probabilistic model is additive (as for the Logit model).

\subsection{Exogenous vs. endogenous dwelling price models}

In the above modelling architecture, the dwelling price can be treated either as a variable exogenous with respect to the decision process of the agents of the study area, or as an endogenous variable. In the one case, it is an attribute of the zones not depending on the locating choices of the agents, but only on exogenous factors such as the accessibility of the zone, the built environment, the parking facilities of the zone, the presence of green spaces, etc. In the other case (endogenous price), the dwelling price is affected by the location choice of the agents and results from the equilibrium among the demand and the supply of floor space available in that zone (supply).

Let " $\mathrm{j}$ " denote the typology of the floor space available in the study area, (e.g. detached dwellings, semi-detached, apartments, shops, sheds,...), and let $X_{\mathrm{j}}(\mathrm{o})$ be the average price of the floor space of type " $\mathrm{j}$ " in the zone "o". An exogenous price model can be formulated as follows:

$X_{\mathrm{j}}(\mathbf{0})=\sum_{\mathrm{n}} \gamma_{\mathrm{n}} \cdot X_{\mathrm{nj}}(\mathrm{o})+\varepsilon$

where the $X_{\mathrm{nj}}(\mathrm{o})$ are the characteristics of the zone, the $\gamma^{\prime} \mathrm{s}$ are parameters to be estimated and the $\varepsilon$ is the error term.

Let $S_{\mathrm{j}}(\mathrm{o})$ be the floor space of the dwelling of typology $\mathrm{j}$ available in the zone "o" and $\delta_{\mathrm{ij}}$ be an index equal to 1 if agent " $\mathrm{i}$ " might be interested in the dwelling of type $j$ (or, equivalently, if the dwelling of type " $\mathrm{j}$ " belongs to the choice set of the agent " $\mathrm{i}$ "), 0 otherwise. Note that the definition of the $\delta_{\mathrm{ij}}$ indexes is not trivial and may depend both on the urban agents considered in the application of the model and on the specific case study. Typically, four types of dwelling stock may be distinguished: "warehouse", "detached/semi-detached house", "single-storey building" and "flat" or "apartment". Some of these typologies are clearly available only for given urban activities: for example a warehouse is typically available only for industrial use or for wholesale commercial activities. On the other hand, some dwelling typologies may be available to different urban agents according to the case study being considered: a single-storey building, for instance, is typically considered available only for retail uses (and thus available only to retailers), but there are some cases (e.g. some historical residential areas) in which it is available also for residential use (see neighbourhoods like Chelsea in London or the Upper-East Side in Manhattan, New York City). In the application presented in the next section, we have considered the two classes of housing stock, i.e. detached/semi-detached, and flat/apartment, available for mixed use. We have not considered either the "warehouse" type given the urban agents whose location choice we are simulating (i.e. residents, service and commercial firms), nor "single-storey buildings", due to a lack of reliable data on the prices of such a typology in our study area. An endogenous price model can be formulated as follows:

$X_{\mathrm{j}}(\mathbf{o})=\gamma_{0} \cdot X_{0 \mathrm{j}}(\mathbf{0})+\gamma_{1} \cdot\left(\frac{\sum_{i} \delta_{\mathrm{ij}} A^{\mathrm{i}}(\mathbf{0})}{S_{\mathrm{j}}(\mathbf{0})}\right)^{\gamma_{2}}+\varepsilon$

where

- $X_{0, j}(0)$ is the hedonic price of the dwellings of typology " $\mathrm{j}$ " in zone "o", depending on the characteristics of the zone;

- the second term represents the ratio between the demand and the supply of dwellings of type " $\mathrm{j}$ " in zone "o";

- $\gamma_{0}, \gamma_{1}$ and $\gamma_{2}$ are parameters to be estimated;

$-\varepsilon$ is the error term.

From the above model specifications it can be seen that in the exogenous price models, the average price of dwellings in a generic zone is given by a multiple regression of several attributes which 
are not influenced by the location decision process of the agents of the study area. On the other hand, in the endogenous price models, the dwelling price depends on the number of agents which chose to locate their activity in the given zone (demand) and on the available floor space in that zone (supply). In other words, they result from the interactions between the agents involved in location decision process in the study area.

\section{Model specification for the metropolitan area of Napoli}

\subsection{The study area}

The study area for the application of the model system presented in the previous section is the metropolitan area of Napoli (South Italy). It includes 88 municipalities, with a total population of 2.4 million residents and about 680,000 jobs. The zoning system adopted consists of 133 zones, most of which represent a single municipality, only the most dense and wide-spread urbanized areas have been split into different zones. For instance, the city of Napoli has been split into 27 zones, Torre Annunziata into 3 zones, and so on. Moreover, the zones have been grouped into territorial "basins", i.e. macro-areas which includes municipalities with a high level of interaction due to administrative, political and historical reasons, e.g. the municipalities belong to the same health district, or to the same justice court, or to the same industrial district, and so on (Table 1).

We have here considered 6 basins (Fig. 2): "Napoli", including the city of Napoli and its hinterland; "Aversa" and "San Giuseppe" two agglomerations of municipalities respectively staying North and West of Napoli; "Torre Annunziata", "Castellammare" and "Peninsula Sorrentina" at the southern end of the Gulf of Napoli. For each basin we have identified an "attractor", i.e. the municipality which has the main number of residents and economic activities and/or which has a leadership role among the other municipalities of the basin (e.g. due to the presence of a justice court, a hospital, etc.).

The population is concentrated in the city of Napoli and along the coast of the Gulf of Napoli. Here we have zones with a population density over 10,000 residents per square kilometre with peaks of 17,000 people/sq-km. The economic activities present the same spatial distribution as the population (Fig. 3): a strong concentration of activities in the city centre of Napoli can be observed, with peaks of density of 10,000 jobs/sq-km.

\subsection{The activities spatial distribution sub-model}

The activity location sub-model has been specified and calibrated for 4 urban-agent classes: the workers of the study area distinguished by income (i.e. the "High" and the "Medium-Low" income workers) and the firms involved in private services and commerce. The location utility function for the generic agent "i", includes the following attributes:
- the commuting generalized travel cost (expressed in hours) between the zone of residence and the workplace zone (note that, as it can be drawn from Table 2, this attribute is included in the location utility of only the classes of workers);

- the passive accessibility with respect to the population of the study area (note that, as it can be drawn from Table 2, this attribute is included in the location utility of only the classes of firms); for further details see Section 4.3;

- the active accessibility of the households to services (e.g. schools, banks, hospitals, ...); for further details see Section 4.3;

- the dwelling stock, distinguished by type: a) apartments, detached and semi-detached houses available for all workers and for the firms involved in services; b) stores on frontage streets and in flats available for service firms (e.g. banks) and commercial firms;

- the dwelling price, expressed in thousands of Euro per square metre (sqm);

- the number of shopping malls in the zone (note that this attribute is included in the location utility of only the classes of firms);

- the number of hospitals in the zones;

- a dummy variables equal to 1 if the zone belongs to the municipality of Napoli, 0 otherwise;

- a dummy variables equal to 1 if the zone belongs to a touristic area, 0 otherwise;

- a dummy variables equal to 1 if the zone belongs to an industrial district, 0 otherwise;

- a dummy variables equal to 1 if the zone is the "attractor" of the basin, 0 otherwise;

- a dummy variable equal to 1 if the zone belongs to a dense urbanized area;

- and finally, six basin specific constants.

The parameters $\beta$ 's of the systematic location utility function have been estimated, starting from an existing model specification (Nuzzolo \& Coppola, 2007) through the fine-tuning of the parameters, in order to match the model predictions in the reference scenario with the census data.

From the values reported in Table 2, it can be noted that the dwelling price parameter in the choice of location is more relevant for the "medium/low-income" class than for the "high-income" one and for firms in "services". Moreover, for "services" accessibility plays a greater role than for commercial activities, whose location, on the other hand, is more affected by the spatial distribution of the population.

From the relative weights of the parameters the trade-off between the attributes can be estimated. For instance it can be deduced that $1 \mathrm{~h}$ reduction in commuting times, for the class of "medium-low income workers", is equivalent to $2.1(=-1.1 /$ $-0.52) \times 10^{3}$ euro per square metre in the dwelling price, i.e.

Table 1

Population and employment in the basins of the study area.

\begin{tabular}{|c|c|c|c|c|c|c|}
\hline Basin & $\begin{array}{l}\text { Number } \\
\text { of zones }\end{array}$ & $\begin{array}{l}\text { Number of } \\
\text { municipalities }\end{array}$ & Population & $\begin{array}{l}\text { Total } \\
\text { employment } \\
\text { (n. of jobs) }\end{array}$ & $\begin{array}{l}\text { Emploment } \\
\text { services } \\
\text { (n. of jobs) }\end{array}$ & $\begin{array}{l}\text { Employment } \\
\text { commerce } \\
\text { (n. of jobs) }\end{array}$ \\
\hline Aversa & 18 & 18 & 174,234 & 37,046 & 11,448 & 6150 \\
\hline Castellammare & 9 & 8 & 102,950 & 26,519 & 6652 & 4729 \\
\hline Napoli & 84 & 42 & $1,809,151$ & 545,375 & 163,807 & 88,687 \\
\hline Pen. Sorrentina & 6 & 6 & 76,053 & 17,756 & 7579 & 3293 \\
\hline San. Giuseppe & 8 & 8 & 123,107 & 28,598 & 5656 & 8201 \\
\hline \multirow[t]{2}{*}{ Torre A. } & 8 & 6 & 129,157 & 28,116 & 6831 & 5455 \\
\hline & 133 & 88 & $2,414,653$ & 683,410 & 201,973 & 116,515 \\
\hline
\end{tabular}




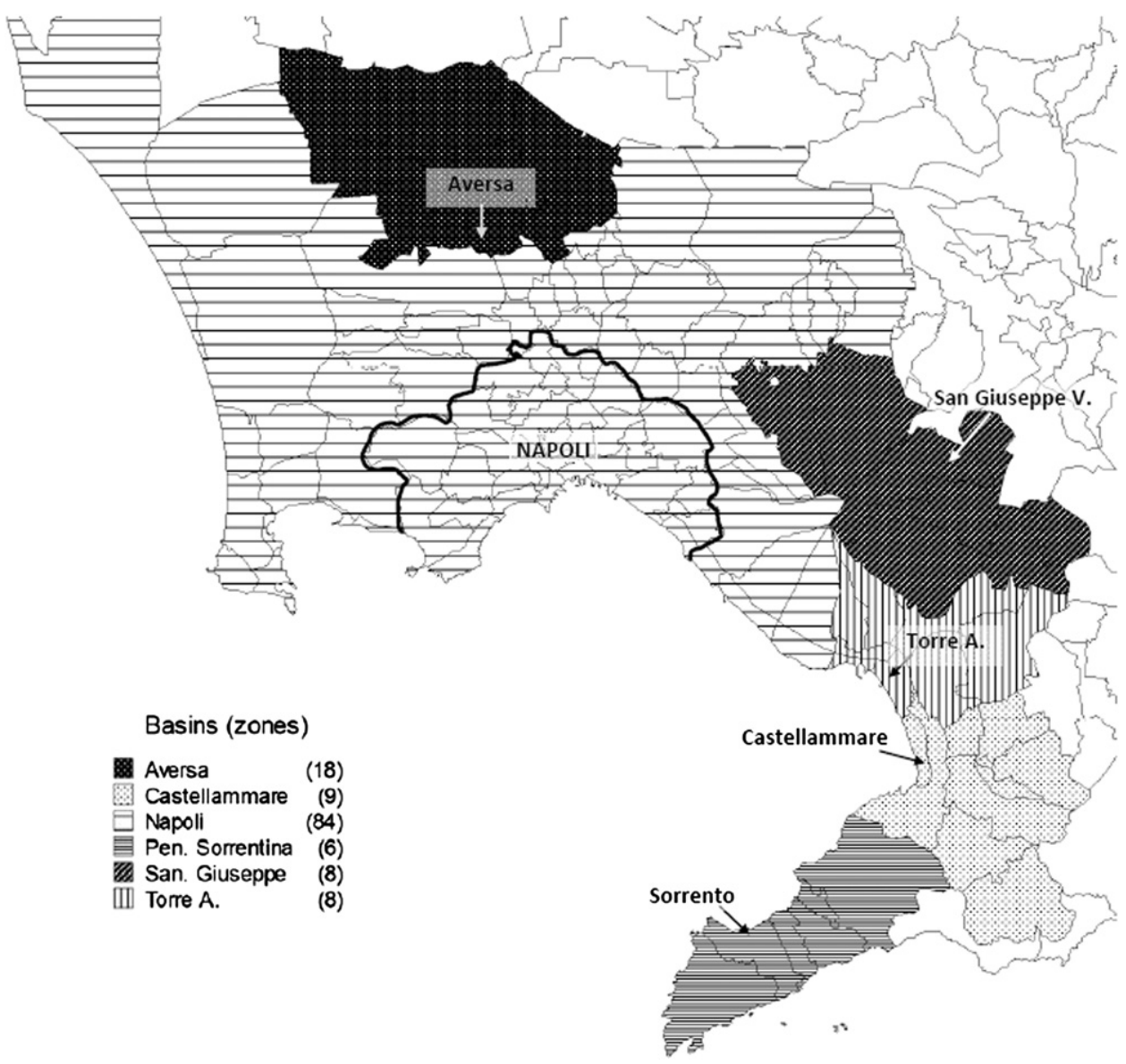

Fig. 2. Basins and relative "attractors" of the study area

a mid-low income worker willingness-to-pay for buying a house closer by $30 \mathrm{~min}$ to the workplace is about 1.050 Euro/sqm. Moreover, it can also be observed that such willingness-to-pay is about one quarter (actually 0.28 ) of the willingness-to-pay for buying a house of the "high-income workers".

\subsection{The accessibility functions}

The accessibilities have been expressed either as the potential of a given zone to be reached by other agents (Passive accessibility) or as the potential to reach the other agents (Active accessibility) from
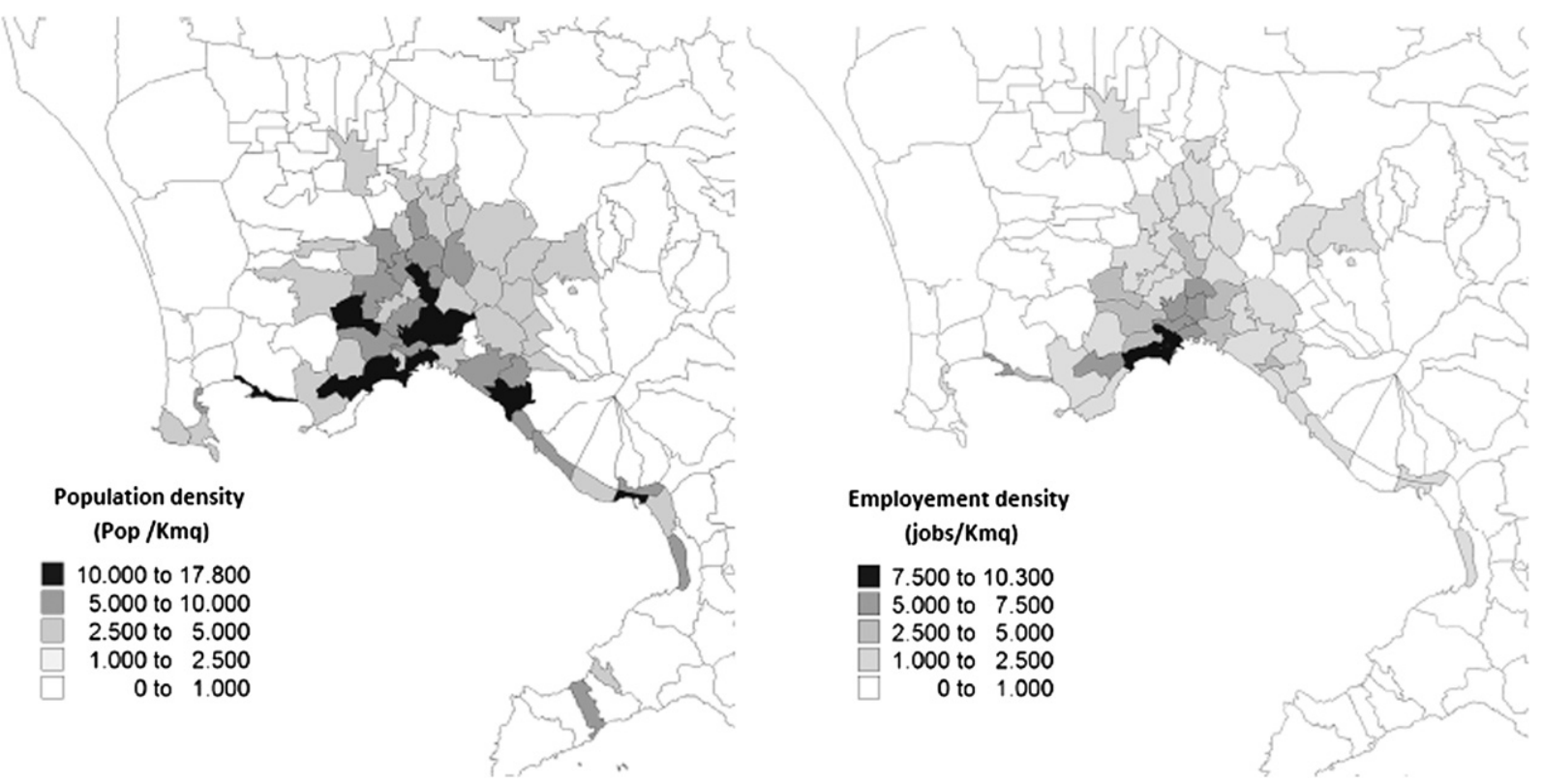

Fig. 3. Population and employment densities. 
Table 2

Coefficients of the location utility function.

\begin{tabular}{|c|c|c|c|c|}
\hline Attribute & $\begin{array}{l}\text { Workers } \\
\text { (high- } \\
\text { income) }\end{array}$ & $\begin{array}{l}\text { Workers } \\
\text { (medium- } \\
\text { low income) }\end{array}$ & $\begin{array}{l}\text { Firms } \\
\text { (service } \\
\text { sector) }\end{array}$ & $\begin{array}{l}\text { Firms } \\
\text { (commerce) }\end{array}$ \\
\hline Commuting cost [hours] & -0.800 & -1.100 & - & - \\
\hline $\begin{array}{l}\text { Passive accessibility } \\
\text { w.r.t to population }\end{array}$ & - & - & 0.025 & 0.015 \\
\hline $\begin{array}{l}\text { Active accessibility } \\
\text { w.r.t .to services }\end{array}$ & 0.002 & 0.003 & - & - \\
\hline Population [thousands] & - & - & 0.350 & 0.500 \\
\hline Jobs in public sectors & - & - & 0.005 & 0.020 \\
\hline $\begin{array}{l}\text { Dwelling price } \\
\text { [103Euro/sqm] }\end{array}$ & -0.105 & -0.520 & -0.055 & \\
\hline Dwelling stock [thousands] & 1.100 & 2.600 & - & 0.002 \\
\hline $\mathrm{N}$. of shopping mall & - & - & 0.450 & 0.650 \\
\hline N. of hospitals & - & - & -0.050 & -0.100 \\
\hline Dummy_tribunal & - & - & 0.300 & 0.400 \\
\hline Dummy turistic area & - & - & 0.400 & 0.200 \\
\hline $\begin{array}{l}\text { Dummy_industrial } \\
\text { district }\end{array}$ & - & - & 0.350 & 0.350 \\
\hline Dummy_urban area & 0.200 & 0.200 & - & - \\
\hline $\begin{array}{l}\text { Dummy_attractor } \\
\text { of the basin }\end{array}$ & - & - & 0.500 & 0.450 \\
\hline $\begin{array}{l}\text { Municipality of } \\
\text { Naples }\end{array}$ & 0.225 & 0.200 & 0.950 & 0.450 \\
\hline Basin_Naples & -0.300 & -0.500 & 0.550 & 0.400 \\
\hline Basin_Aversa & -0.400 & -0.300 & 0.200 & -0.200 \\
\hline Basin_Torre A. & -0.300 & -0.200 & 0.050 & 0.100 \\
\hline Basin_Castellammare & -0.050 & 0.300 & 0.050 & -0.050 \\
\hline Basin_San Giuseppe & -0.200 & -0.050 & -0.050 & 0.400 \\
\hline Basin_Pen. Sorrentina & 0.600 & 0.700 & 0.550 & 0.050 \\
\hline
\end{tabular}

a given zone. For instance, the active accessibility of households to services, has been calculated as:

$\operatorname{Acc}$ active $(\mathbf{o})=\sum_{d} W(\mathrm{~d})^{\alpha_{1}} \cdot \exp \left(\alpha_{2} \cdot C(\mathbf{o}, \mathbf{d})\right)$

where

- $W(\mathrm{~d})$ is the number of services (expressed in thousands) in the zone "d";

- $C(\mathrm{o}, \mathrm{d})$ is the generalized travel cost between zones "o" and " $\mathrm{d}$ ";

- $\alpha_{1}$ and $\alpha_{2}$ are estimated parameters respectively equal to 0.85 and 1.25 (Nuzzolo \& Coppola, 2007).

Moreover, the passive accessibility of the firms to the population, has been calculated as:

$\operatorname{Acc}$ _passive $(\mathrm{d})=\sum_{o} \mathrm{HH}\left(o^{\alpha_{3}} \cdot \exp \left(\alpha_{4} \cdot \mathrm{C}(\mathrm{o}, \mathrm{d})\right)\right.$

where

- $\mathrm{HH}(\mathrm{o})$ is the number of households (expressed in thousands) in the zone "o";

- $C(\mathrm{o}, \mathrm{d})$ is the generalized travel cost between zones "o" and " $\mathrm{d}$ ";

- $\alpha_{3}$ and $\alpha_{4}$ are two estimated parameters, respectively equal to

0.72 and 1.14 (Nuzzolo \& Coppola, 2007).

\subsection{The dwelling price functions}

Based on a survey carried out through the real-estate agencies in the study area, the average prices of the dwellings in the zones of the study area, available either for residential use by the households or for services activities by the firms (i.e. the above defined typology "a", consisting of the apartments, the detached and semidetached houses) have been estimated (Fig. 4).

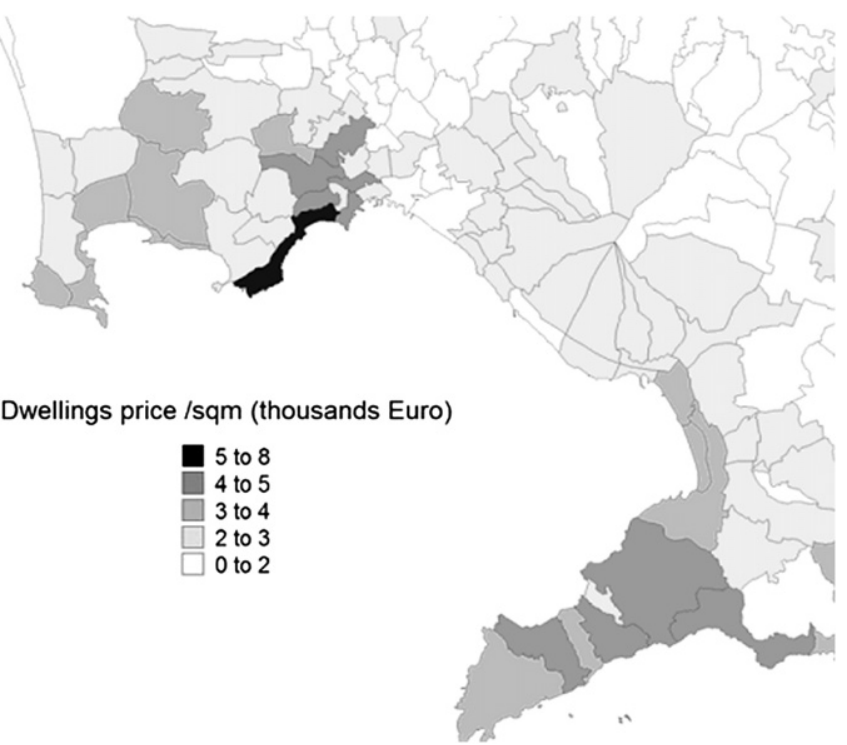

Fig. 4. Average dwelling price per square metre in the zones of the study area.

Based on such observations, two different dwelling price functions have been estimated. The exogenous price location function is specified as follows:

$X(\mathbf{o})=\gamma_{0} \cdot X_{0}(\mathbf{0})+\gamma_{1} \cdot \operatorname{Acc}$ ppassive $(\mathbf{o})$

where:

- the $X(0)$ is the average dwelling price per square metre (expressed in thousands of euro) in zone "o";

- the $X_{0}(0)$ is the dwellings hedonic price per square metre in zone "o", depending on the characteristic of the zone such as the quality of the built environment, the presence of green spaces, etc.;

- Acc_passive(o) is the passive accessibility of the zone "o";

- $\gamma_{0}$ and $\gamma_{1}$ are parameters respectively equal to 0.857 and to 0.0032 .

The endogenous price location function is specified as follows:

$X(\mathbf{o})=\gamma_{0} \cdot X_{0}(\mathbf{o})+\gamma_{1} \cdot\left(\frac{\mathrm{HH}(\mathrm{o})+\mathrm{SF}(\mathbf{o})}{\operatorname{Stock}_{\mathrm{a}}(\mathbf{0})}\right)^{\gamma_{2}}$

where:

- $X(0)$ and $X_{0}(0)$ are the average dwelling prices per square metre defined in section 3.1 ;

- $\mathrm{HH}(\mathrm{o})$ is the number of households in zone "o";

- $\mathrm{SF}(\mathrm{o})$ is the number of firms involved in services in the zone "o";

- $\operatorname{Stock}_{\mathrm{a}}(\mathrm{o})$ the number of dwellings of typology a (above defined) in the generic zone "o";

- $\gamma_{0}, \gamma_{1}$ and $\gamma_{2}$ the estimated parameters equal to $0.717,4.1$ and 1.8 .

\subsection{Model validation}

To validate the model, we have made a simulation of the reference scenario (i.e. year 1981) and analysed the differences between the model predictions and the "observed" census data. The differences in percentage, at the level of a single basin, are less than $8 \%$ (Table 3 ). 
Table 3

Reference scenario (year 2001) model prediction vs. census data.

\begin{tabular}{|c|c|c|c|c|c|c|c|c|}
\hline \multirow[t]{2}{*}{ Basin } & \multicolumn{2}{|c|}{ High income [workers] } & \multirow[b]{2}{*}{ Diff. } & \multirow[b]{2}{*}{ \% Diff. } & \multicolumn{2}{|c|}{$\begin{array}{l}\text { Medium-Low income } \\
\text { [workers] }\end{array}$} & \multirow[b]{2}{*}{ Diff. } & \multirow[b]{2}{*}{ \% Diff. } \\
\hline & Census & Model & & & Census & Model & & \\
\hline Aversa & 3,197 & 3,262 & 65 & $2.0 \%$ & 46,084 & 49,018 & 2,934 & $6.4 \%$ \\
\hline Castellammare & 2,088 & 2,165 & 77 & $3.7 \%$ & 29,806 & 31,367 & 1,561 & $5.2 \%$ \\
\hline Napoli & 44,454 & 45,084 & 630 & $1.4 \%$ & 517,353 & 516,436 & 9,083 & $1.8 \%$ \\
\hline Pen. Sorrentina & 2,428 & 2,445 & 17 & $0.7 \%$ & 26,513 & 25,364 & $-1,149$ & $-4.3 \%$ \\
\hline San Giuseppe & 2,691 & 2,765 & 74 & $2.7 \%$ & 36,918 & 39,772 & 2,854 & $7.7 \%$ \\
\hline \multirow[t]{2}{*}{ Torre Annunziata } & 2,447 & 2,592 & 145 & $5.9 \%$ & 37,238 & 39,828 & 2,590 & $7.0 \%$ \\
\hline & 57,305 & 58,313 & 1,008 & $1.8 \%$ & 693,912 & 701,785 & 7,873 & $1.1 \%$ \\
\hline \multirow[t]{2}{*}{ Basin } & \multicolumn{2}{|c|}{ Private Services [jobs] } & & & \multicolumn{2}{|c|}{ Commerce [jobs] } & & \\
\hline & Census & Model & Diff. & \% Diff. & Census & Model & Diff. & \% Diff. \\
\hline Aversa & 11,448 & 11,444 & -4 & $0.0 \%$ & 6,150 & 6,252 & 102 & $1.7 \%$ \\
\hline Castellammare & 6,652 & 6,231 & -421 & $-6.3 \%$ & 4,729 & 4,496 & -233 & $-4.9 \%$ \\
\hline Napoli & 163,807 & 165,381 & 1574 & $1.0 \%$ & 88,687 & 89,823 & 1136 & $1.3 \%$ \\
\hline Pen. Sorrentina & 7,579 & 7,265 & -314 & $-4.1 \%$ & 3,293 & 3,284 & -9 & $-0.3 \%$ \\
\hline San Giuseppe & 5,656 & 5,714 & 58 & $1.0 \%$ & 8,201 & 7,900 & -301 & $-3.7 \%$ \\
\hline \multirow[t]{2}{*}{ Torre Annunziata } & 6,831 & 6,340 & -491 & $-7.2 \%$ & 5,455 & 5,196 & -259 & $-4.7 \%$ \\
\hline & 201,973 & 202,375 & 402 & $0.2 \%$ & 116,515 & 116,951 & 436 & $0.4 \%$ \\
\hline
\end{tabular}
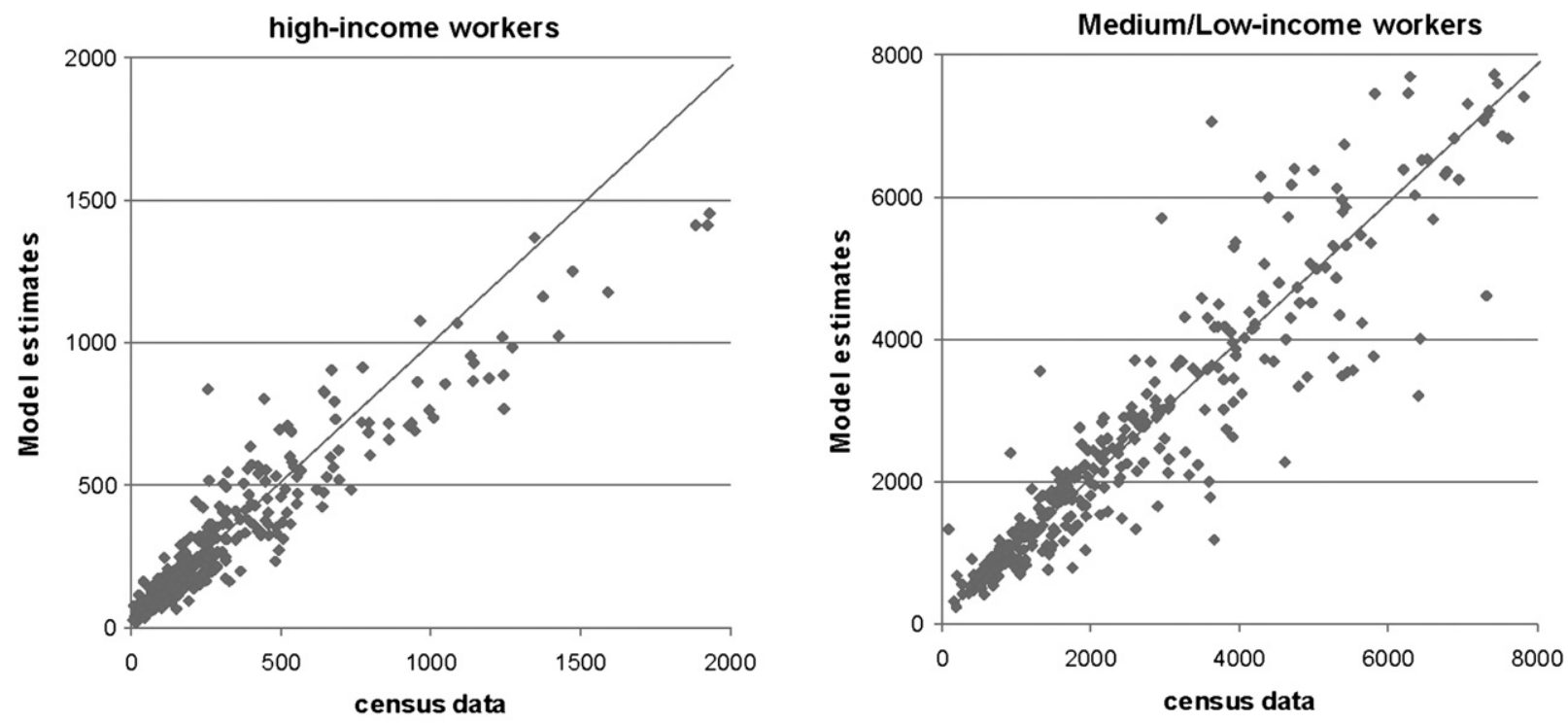

Private Services
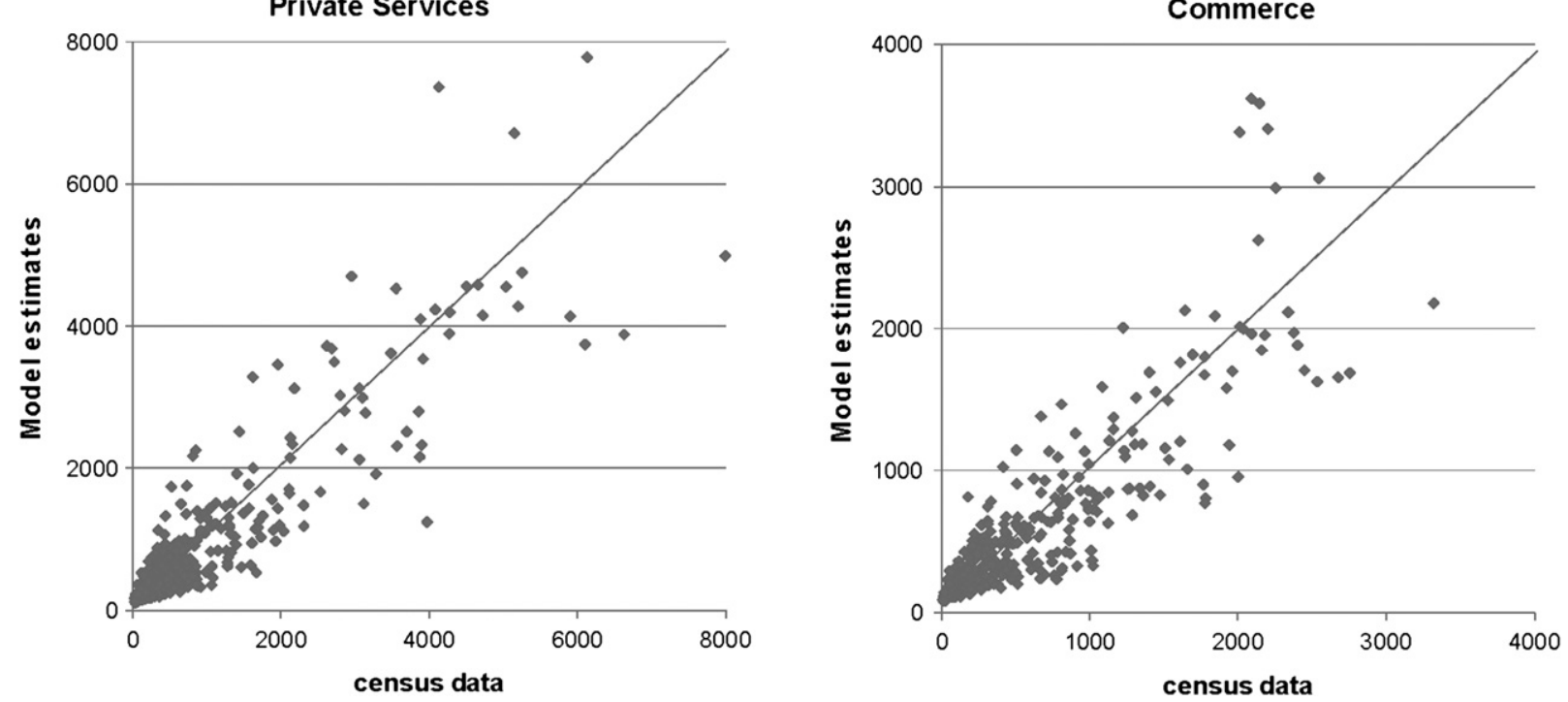

Fig. 5. Reference scenario (year 1981) model's predictions ( $y$-axis) vs. census data ( $x$-axis). 


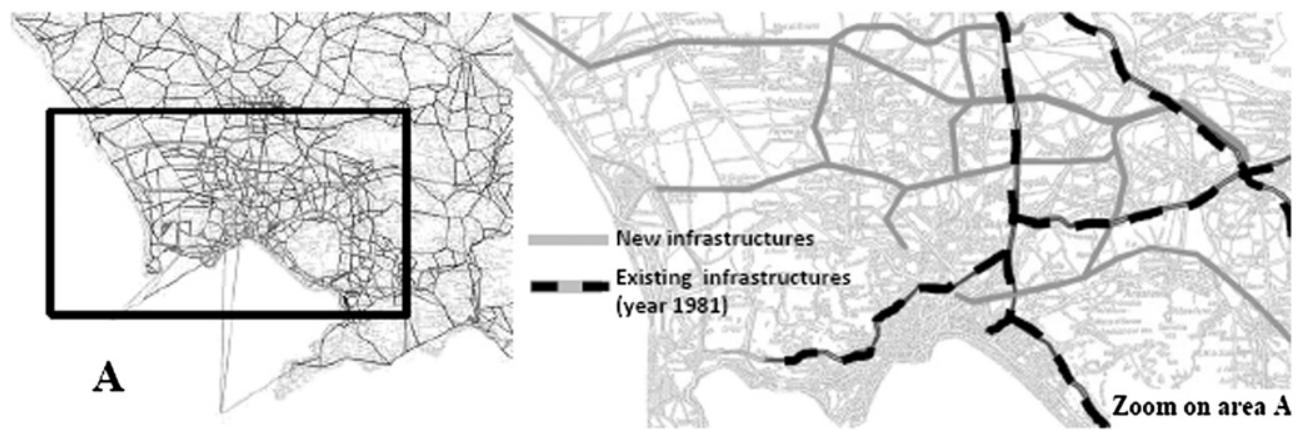

Fig. 6. The new road infrastructures in the "forecasting" scenario (dashed lines represent existing highways in the reference scenario, i.e. year 1981).

At the level of the single zone, the overall matching between model predictions and census data is good. However, in some zones a peak in percentage differences (model vs. census) of over $50 \%$ can be observed (Fig. 5).

To avoid such peaks distorting the result of applying the model to future scenarios, we have adopted the "pivot-point" technique (Manheim, 1979) to predict the number of agents of class " $i$ " in the zone "o", $A^{\mathrm{i}}(\mathrm{o})$ :

$A^{\mathrm{i}}(\mathrm{o})=\frac{A_{\text {future }}^{\mathrm{i}}}{A_{\text {ref }}^{\mathrm{i}}} \cdot A_{\text {census_ref }}^{\mathrm{i}}$

where:

- $A^{i}$ future is the number of agents of class " $i$ " in the zone "o", predicted by the model in the future scenario;

- $A_{\text {ref }}^{\mathrm{i}}$ is the number of agents of class " $\mathrm{i}$ " in the zone "o", predicted by the model in the reference scenario;

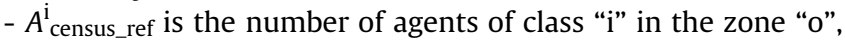
observed by the Census in the reference scenario.

\subsection{Model forecasting}

To test the forecasting capability of the model we have conducted an application of the model system to the 2001 scenario, using as reference a transportation scenario dated back to year 1981 (i.e. a "backcasting" analysis). In other words, we have applied the model as if we were in the year 1981 and wanted to predict the spatial distribution of the activities in the study area on a 20-year long time horizon (i.e. year 2001). Then, we have compared the forecasting results in year 2001 with the available Census data of that year.

In building up the "forecasting" scenario (2001), it became apparent that the main changes to the transportation system took place on the road network between the years 1985 and 1990. This can be explained by the fact that in year 1980 a major earthquake devastated the whole Region, particularly the area around Napoli and Avellino. Consequently a wide reconstruction plan allowed the renovation and partly the reconstruction of the regional road network, particularly in the area North of Napoli (see Fig. 6).

In these applications of the model system, two different dwelling price models have been tested: exogenous vs. endogenous dwelling price models. The results we obtained show a significant difference in the forecast population pattern. In fact, using the exogenous price model, it can be observed (see Fig. 7), on the one hand, an overall increase of population in the zones with the greater increase of accessibility (i.e. the area North of Napoli, some zones of Napoli and the zones along the northern coast of the Gulf); on the other hand, a decrease of population in the zones of the Peninsula Sorrentina, which are the zones less affected by the changes of accessibility due to the new roads.
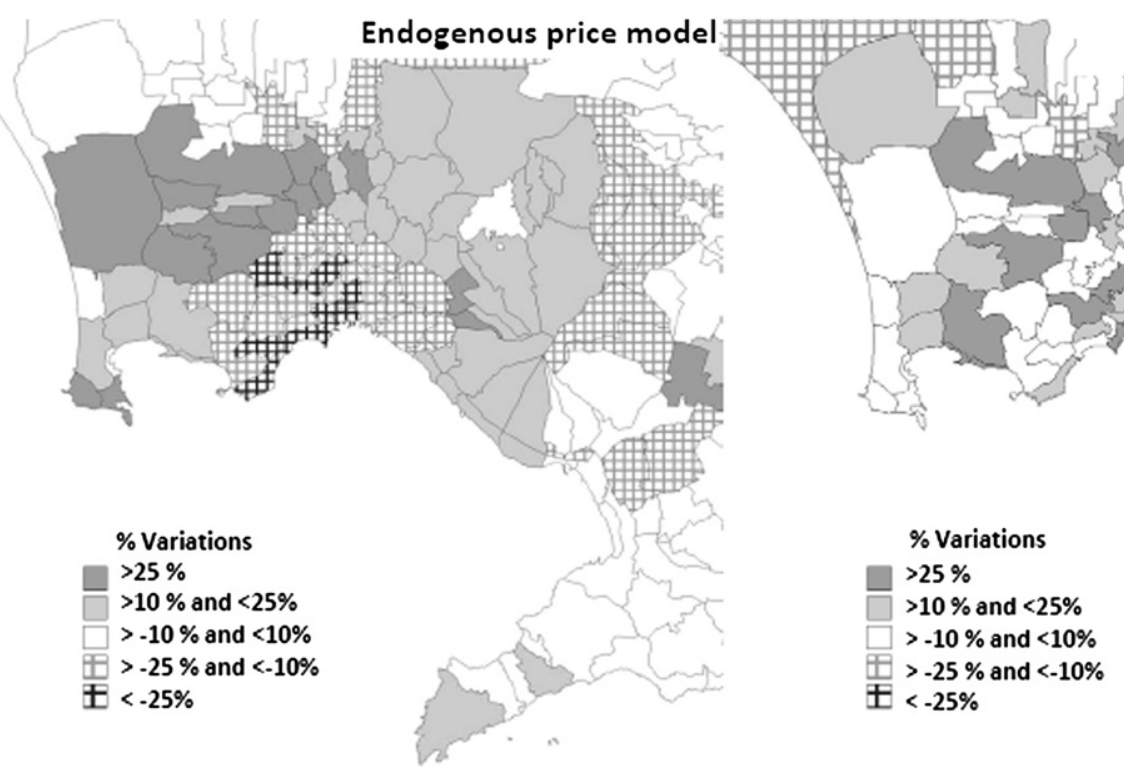

Exogenous price model

Fig. 7. Population variations predicted by endogenous and exogenous dwellings price models. 


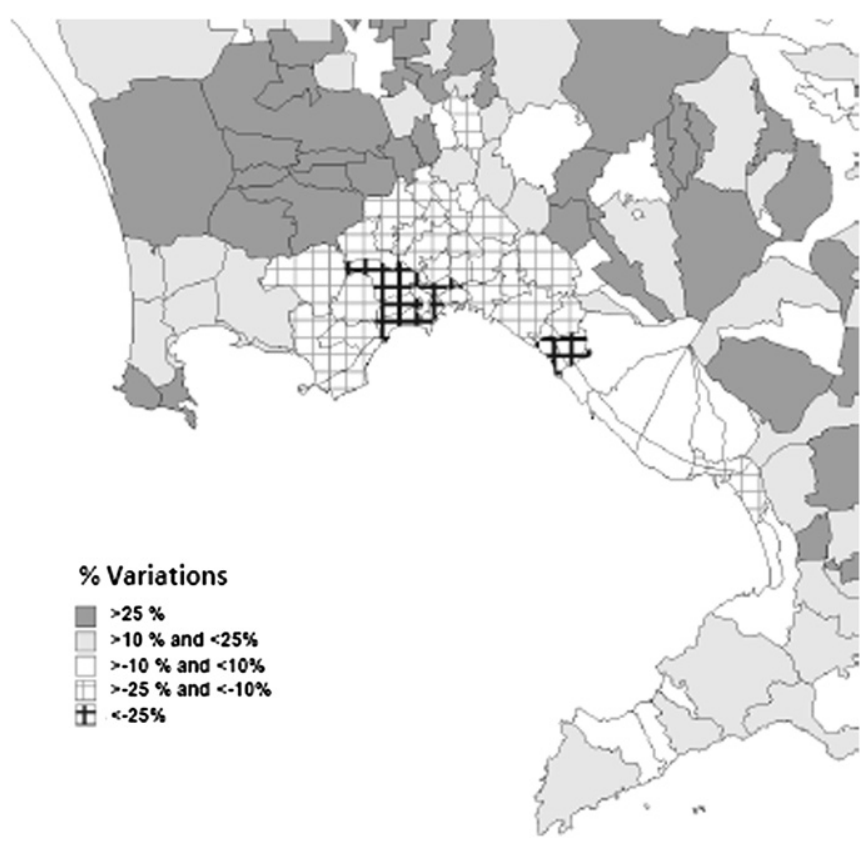

Fig. 8. Population variations between 2001 and 1981 in the study area, by Census data.

The endogenous price model predict a different population pattern with a significant decrease of population in the zones of Napoli and an increase in the zones North of the study area (Fig. 7). Such differences can be explained by the fact that the increase of accessibility in Napoli does attract more urban agents which in turn induces an increase of the dwelling price, due to limited availability of dwelling stock. The competition of different agents for acquiring the floor space available in such zones has resulted in a replacement of households with firms, the former having a lower willingness-to-pay than the latter. This resulted in the migration of the population in the area of new development located in the North of the study area.

If we compare the results of the simulation using the two different models with the observed percentage changes in population from the Census (Fig. 8), we can conclude that the endogenous dwelling price model performs better than the exogenous one in forecasting the population pattern. This is due to the fact that the former does explicitly simulate the interactions among the different urban agents.

\section{Conclusions}

In this paper we have presented a modelling framework to forecast the evolution of the spatial distribution pattern of the activities in a metropolitan area, based on the simulation of the location choices of multiple agents of the urban system (i.e. the workers disaggregated by income, the firms by economic sector). The parameters of the overall model system have been calibrated for the metropolitan area of Napoli by fine-tuning of the parameters of an existing model specification.

The calibrated model system reproduces, with a good level of approximation, the spatial distribution of the population and of the firms within the study area.

Two different approaches to simulate the interactions among the location choice of the agents and the dwellings price have been tested: the "exogenous price" approach and the "endogenous price" approach.

A "backcasting" application of the model system using the above two approaches has shown that the endogenous dwelling price model outperforms the exogenous one. Therefore, we can conclude that in a dense urbanized area, where the dwelling market is saturated, it is necessary to take explicitly into consideration the interactions among the different urban agents, and the effects of such interactions on the dwelling price, in order to correctly forecast the evolution of the land use pattern.

\section{References}

Alonso, W. (1964). Location and land use. Cambridge, MA: Harvard University Press. Anas, A., \& Xu, R. (1999). Congestion, land use, and job dispersion: a general equilibrium model. Journal of Urban Economics, 45, 451-473.

Cascetta, E. (2009). Transportation systems analysis: Models and applications (2nd ed.). Springer.

Chang, J., \& Mackett, R. (2005). A bi-level model of the relationship between transport and residential location. Transportation Research B, 40, 123-146.

Clarke, K. C., Hoppen, S., \& Gaydos, L. (1997). A self-modifying cellular automaton model of historical urbanization in the San Francisco Bay area. Environment and Planning B: Planning and Design, 24, 247-261.

De la Barra, T. (1989). Integrated land use and transport modelling: Decision chains and hierarchies. Cambridge, UK: Cambridge University Press.

De Palma, A., Motamedi, K., Picard, N., \& Waddell, P. (2006). A model of residential location choice with endogenous dwellings prices and traffic or the Paris region. European Transport, 3, 67-82.

Herbert, J., \& Stevens, B. (1960). A model of the distribution of residential activity in urban areas. Journal of Regional Science, 2, 21-36.

Manheim, M. L. (1979). Fundamentals of transportation system analysis. Cambridge, MA: MIT Press.

Nuzzolo, A., \& Coppola, P. (2007). Accessibility and socioeconomic activities location. In Proceedings of the European transport conference 2007. London, UK: Association for European Transport and Contributors.

Waddell, P. (2002). UrbanSim: modeling urban development for land use, transportation and environmental planning. Journal of the American Planning Association, $68,297-314$

Waddell, P., Borning, A., Noth, M., Freier, N., Becke, M., \& Ulfarsson, G. (2003). Microsimulation of urban development and location choices: design and implementation of UrbanSim. Networks and Spatial Economics, 3, 43-67.

Zhou, B., \& Kockelman, K. (2008). Microsimulation of residential land development and household location choices: bidding for land in Austin, Texas. Transportation Research Record, 2077, 106-112. 